\title{
Sobolev-Besov spaces of measurable functions
}

by

\author{
HANS Triebel (Jena)
}

Abstract. The paper deals with spaces $\mathbf{L}_{p}^{s}\left(\mathbb{R}^{n}\right)$ of Sobolev type where $s>0$, $0<p \leq \infty$, and their relations to corresponding spaces $\mathbf{B}_{p, q}^{s}\left(\mathbb{R}^{n}\right)$ of Besov type where $s>0,0<p \leq \infty, 0<q \leq \infty$, in terms of embedding and real interpolation.

\section{Introduction. Let}

$$
\left(\Delta_{h}^{1} f\right)(x)=f(x+h)-f(x), \quad\left(\Delta_{h}^{k+1} f\right)(x)=\Delta_{h}^{1}\left(\Delta_{h}^{k} f\right)(x)
$$

with $h \in \mathbb{R}^{n}, x \in \mathbb{R}^{n}, k \in \mathbb{N}$, be the usual differences of complex-valued Lebesgue measurable functions in $\mathbb{R}^{n}$. Let $0<p, q \leq \infty$ and $0<s<k \in \mathbb{N}$. Then $\mathbf{B}_{p, q}^{s}\left(\mathbb{R}^{n}\right)$ is the collection of all $f \in L_{p}\left(\mathbb{R}^{n}\right)$ such that

$$
\left\|f\left|\mathbf{B}_{p, q}^{s}\left(\mathbb{R}^{n}\right)\left\|_{k}=\right\| f\right| L_{p}\left(\mathbb{R}^{n}\right)\right\|+\left(\int_{0}^{1} t^{-s q} \sup _{|h| \leq t}\left\|\Delta_{h}^{k} f \mid L_{p}\left(\mathbb{R}^{n}\right)\right\|^{q} \frac{d t}{t}\right)^{1 / q}
$$

is finite (these are equivalent quasi-norms for all $k>s$ ). If $s>n \max (0$, $1 / p-1)$ then $\mathbf{B}_{p, q}^{s}\left(\mathbb{R}^{n}\right)$ coincides with the Fourier-analytically defined spaces $B_{p, q}^{s}\left(\mathbb{R}^{n}\right)$ (appropriately interpreted). If $1<p<\infty$ then the classical Sobolev spaces $W_{p}^{1}\left(\mathbb{R}^{n}\right)$ with

$$
\left\|f\left|W_{p}^{1}\left(\mathbb{R}^{n}\right)\|=\| f\right| L_{p}\left(\mathbb{R}^{n}\right)\right\|+\sum_{j=1}^{n}\left\|\frac{\partial f}{\partial x_{j}} \mid L_{p}\left(\mathbb{R}^{n}\right)\right\|
$$

can be equivalently normed by

$$
\left\|f\left|W_{p}^{1}\left(\mathbb{R}^{n}\right)\|\sim\| f\right| L_{p}\left(\mathbb{R}^{n}\right)\right\|+\sup _{0<|h| \leq t<1} t^{-1}\left\|\Delta_{h}^{1} f \mid L_{p}\left(\mathbb{R}^{n}\right)\right\|,
$$

[30, Proposition 3, p. 139] or [39, pp. 45-46]. One may also consult [24, Section 6, pp. 226-227] for a very early attempt to extend (1.3) to $0<p<1$. According to B. Bojarski and P. Hajłasz, [8] based on [4], the space $W_{p}^{1}\left(\mathbb{R}^{n}\right)$, $1<p<\infty$, can also be described as the collection of all $f \in L_{p}\left(\mathbb{R}^{n}\right)$ such

2010 Mathematics Subject Classification: Primary 46E35.

Key words and phrases: Sobolev spaces, Besov spaces, embedding, real interpolation. 
that there exists a function $0 \leq g \in L_{p}\left(\mathbb{R}^{n}\right)$ with

$$
|f(x)-f(y)| \leq|x-y|(g(x)+g(y)), \quad x, y \in \mathbb{R}^{n} \text { a.e. }
$$

Furthermore,

$$
\left\|f\left|W_{p}^{1}\left(\mathbb{R}^{n}\right)\|\sim\| f\right| L_{p}\left(\mathbb{R}^{n}\right)\right\|+\inf \left\|g \mid L_{p}\left(\mathbb{R}^{n}\right)\right\|
$$

where the infimum is taken over all $g$ with 1.4 ) (where the Hardy-Littlewood maximal function $g \sim M f$ is an optimal choice). Characterisations of type (1.4), (1.5) in terms of variable Lipschitz coefficients $g$ have been extended in [4, 5] and in the recent papers [2, 3] to higher order Sobolev spaces $W_{p}^{k}\left(\mathbb{R}^{n}\right)$, $1<p<\infty, k \in \mathbb{N}$. In [8] the observation (1.4), 1.5) was taken as a point of departure to introduce Sobolev spaces $W_{p}^{1}(X), 1<p \leq \infty$, on abstract metric spaces $X$. Let $0<s<1$ and $1 \leq p, q<\infty$. If we modify (1.1) with $k=1$ (first differences) appropriately, the corresponding equivalent norms in $B_{p, q}^{s}\left(\mathbb{R}^{n}\right)=\mathbf{B}_{p, q}^{s}\left(\mathbb{R}^{n}\right)$ have direct counterparts on metric spaces $X$ resulting in respective Besov spaces $B_{p, q}^{s}(X)$. Under some additional assumptions ( $X$ is furnished with a doubling measure supporting suitable Poincaré inequalities) the real interpolation

$$
\left(L_{p}(X), W_{p}^{1}(X)\right)_{\theta, q}=B_{p, q}^{\theta}(X), \quad 0<\theta<1,
$$

$1<p, q<\infty$, is one of the main assertions of the recent paper [7].

The following is motivated by $(1.3)-(1.5)$, its indicated generalisations to higher order Sobolev spaces and (1.6). Let $0<p \leq \infty, s>0$ and $k \in \mathbb{N}$ with $s \leq k$. Then $\mathbf{L}_{p}^{s}\left(\mathbb{R}^{n}\right)^{k}$ is the collection of all $f \in L_{p}\left(\mathbb{R}^{n}\right)$ for which there exists a function $0 \leq g \in L_{p}\left(\mathbb{R}^{n}\right)$ such that for all $h \in \mathbb{R}^{n}, 0<|h| \leq 1$,

$$
|h|^{-s}\left|\Delta_{h}^{k} f(x)\right| \leq \sum_{l=0}^{k} g(x+l h), \quad \text { a.e. in } \mathbb{R}^{n} .
$$

Furthermore,

$$
\left\|f\left|\mathbf{L}_{p}^{s}\left(\mathbb{R}^{n}\right)^{k}\|=\| f\right| L_{p}\left(\mathbb{R}^{n}\right)\right\|+\inf \left\|g \mid L_{p}\left(\mathbb{R}^{n}\right)\right\|,
$$

where the infimum is taken over all $g$ with $(1.7)$. These are quasi-Banach spaces. If $s=k \in \mathbb{N}$ then

$$
\mathbf{W}_{p}^{k}\left(\mathbb{R}^{n}\right)=\mathbf{L}_{p}^{k}\left(\mathbb{R}^{n}\right)^{k}, \quad 0<p \leq \infty, k \in \mathbb{N},
$$

are spaces of Sobolev type related to the above construction $1.3-(1.5)$ and the indicated generalisations to higher order Sobolev spaces $W_{p}^{k}\left(\mathbb{R}^{n}\right)$. If $0<s<k \in \mathbb{N}$ then $\mathbf{L}_{p}^{s}\left(\mathbb{R}^{n}\right)^{k}$ seems to be nearer to $\mathbf{B}_{p, \infty}^{s}\left(\mathbb{R}^{n}\right)$. Let $\operatorname{Lip}^{k}\left(\mathbb{R}^{n}\right)$ be suitable Lipschitz spaces of order $k$ and $\mathcal{C}^{k}\left(\mathbb{R}^{n}\right)=B_{\infty, \infty}^{k}\left(\mathbb{R}^{n}\right)$ be the corresponding Zygmund spaces (details may be found below). It is the main aim of this paper to prove the following assertion. 
Theorem. (i) Let $0<s \leq k \in \mathbb{N}$ and $0<p \leq \infty$. Then

$$
\mathbf{B}_{p, \min (p, 1)}^{s}\left(\mathbb{R}^{n}\right) \hookrightarrow \mathbf{L}_{p}^{s}\left(\mathbb{R}^{n}\right)^{k} \hookrightarrow \mathbf{B}_{p, \infty}^{s}\left(\mathbb{R}^{n}\right)
$$

(continuous embeddings).

(ii) Let $0<s \leq k \in \mathbb{N}, 0<p \leq \infty, 0<\theta<1$ and $0<q \leq \infty$. Then

$$
\left(L_{p}\left(\mathbb{R}^{n}\right), \mathbf{L}_{p}^{s}\left(\mathbb{R}^{n}\right)^{k}\right)_{\theta, q}=\mathbf{B}_{p, q}^{s \theta}\left(\mathbb{R}^{n}\right)
$$

(real interpolation).

(iii) Let $k \in \mathbb{N}$. If $1<p<\infty$ then

$$
\mathbf{W}_{p}^{k}\left(\mathbb{R}^{n}\right)=W_{p}^{k}\left(\mathbb{R}^{n}\right) .
$$

Furthermore,

$$
\mathbf{W}_{\infty}^{k}\left(\mathbb{R}^{n}\right)=\operatorname{Lip}^{k}\left(\mathbb{R}^{n}\right) \hookrightarrow \mathcal{C}^{k}\left(\mathbb{R}^{n}\right), \quad \operatorname{Lip}^{k}\left(\mathbb{R}^{n}\right) \neq \mathcal{C}^{k}\left(\mathbb{R}^{n}\right) .
$$

In Section 2 we collect definitions, explanations and some prerequisites. The above theorem will be proved in Section 3 . In Section 4 we add some comments.

All arguments in this paper are rather straightforward with the exception of the left-hand embedding of (1.8), which is based on subatomic decompositions in the spaces $\mathbf{B}_{p, q}^{s}\left(\mathbb{R}^{n}\right)$. It might be considered as a second aim of this paper to demonstrate that building blocks in function spaces are useful also in the above context.

\section{Definitions and prerequisites}

2.1. Definitions. We use standard notation. Let $\mathbb{R}^{n}$ be Euclidean $n$ space, $n \in \mathbb{N}$ (natural numbers) and $\mathbb{N}_{0}=\mathbb{N} \cup\{0\}$. Let $\mathbb{C}$ be the complex plane. Furthermore, $L_{p}\left(\mathbb{R}^{n}\right)$ with $0<p \leq \infty$ is the standard quasi-Banach space with respect to the Lebesgue measure, quasi-normed by

$$
\left\|f \mid L_{p}\left(\mathbb{R}^{n}\right)\right\|=\left(\int_{\mathbb{R}^{n}}|f(x)|^{p} d x\right)^{1 / p}
$$

with the obvious modification if $p=\infty$. Let $\mathbf{M}\left(\mathbb{R}^{n}\right)$ be the collection of the equivalence classes of all almost everywhere finite complex-valued functions with respect to the Lebesgue measure in $\mathbb{R}^{n}$. This linear space, furnished with the convergence in measure, can be converted into a complete metric space. A short description may be found in [36, p. 19] where we referred for more details to [20, Section I,5]. One may consider $\mathbf{M}\left(\mathbb{R}^{n}\right)$ as the largest space covering everything what appears, including the definition of the spaces $\mathbf{B}_{p, q}^{s}\left(\mathbb{R}^{n}\right), \mathbf{L}_{p}^{s}\left(\mathbb{R}^{n}\right)^{k}$, and the convergence of series. (It is the substitute of $D^{\prime}\left(\mathbb{R}^{n}\right)$ and $S^{\prime}\left(\mathbb{R}^{n}\right)$ in the context of distributionally defined function spaces.) But for our purpose it is sufficient to remark that the convergence in the quasi-Banach spaces $L_{p}\left(\mathbb{R}^{n}\right), 0<p \leq \infty$, is stronger than in $\mathbf{M}\left(\mathbb{R}^{n}\right)$, 
[36. p. 19]. For $f \in \mathbf{M}\left(\mathbb{R}^{n}\right), k \in \mathbb{N}$ and $h \in \mathbb{R}^{n}$ let as usual

$$
\left(\Delta_{h}^{1} f\right)(x)=f(x+h)-f(x), \quad\left(\Delta_{h}^{k+1} f\right)(x)=\Delta_{h}^{1}\left(\Delta_{h}^{k} f\right)(x), \quad x \in \mathbb{R}^{n},
$$

be the iterated differences in $\mathbb{R}^{n}$. Let $0<p, q \leq \infty$ and $0<s<k \in \mathbb{N}$. Then $\mathbf{B}_{p, q}^{s}\left(\mathbb{R}^{n}\right)$ is the collection of all $f \in L_{p}\left(\mathbb{R}^{n}\right)$ (or likewise $f \in \mathbf{M}\left(\mathbb{R}^{n}\right)$ ) such that

$$
\left\|f\left|\mathbf{B}_{p, q}^{s}\left(\mathbb{R}^{n}\right)\left\|_{k}=\right\| f\right| L_{p}\left(\mathbb{R}^{n}\right)\right\|+\left(\int_{0}^{1} t^{-s q} \sup _{|h| \leq t}\left\|\Delta_{h}^{k} f \mid L_{p}\left(\mathbb{R}^{n}\right)\right\|^{q} \frac{d t}{t}\right)^{1 / q}
$$

is finite (with the usual modification if $q=\infty$ ). These spaces have some history. Relevant comments and references may be found in [34, pp. 387-389]. In particular, $\mathbf{B}_{p, q}^{s}\left(\mathbb{R}^{n}\right)$ are quasi-Banach spaces which are independent of $k \in \mathbb{N}$ with $s<k$ (equivalent quasi-norms). Some more specific properties, needed later on, are shifted to the next section. We only mention that

$$
\mathbf{B}_{p, q}^{s}\left(\mathbb{R}^{n}\right)=B_{p, q}^{s}\left(\mathbb{R}^{n}\right) \quad \text { if } 0<p, q \leq \infty \text { and } s>n \max (0,1 / p-1)
$$

(appropriately interpreted), where $B_{p, q}^{s}\left(\mathbb{R}^{n}\right)$ are the well-known Fourier-analytically defined spaces, including the classical Besov spaces with $p, q \geq 1$. Let $1 \leq p \leq \infty$ and $k \in \mathbb{N}$. Then $W_{p}^{k}\left(\mathbb{R}^{n}\right)$ are the well-known Sobolev spaces collecting all $f \in L_{p}\left(\mathbb{R}^{n}\right)$ such that

$$
\left\|f\left|W_{p}^{k}\left(\mathbb{R}^{n}\right)\left\|=\sum_{|\alpha| \leq k}\right\| D^{\alpha} f\right| L_{p}\left(\mathbb{R}^{n}\right)\right\|
$$

is finite. Here, as usual,

$$
D^{\alpha} f=\frac{\partial^{|\alpha|} f}{\partial x_{1}^{\alpha_{1}} \ldots \partial x_{n}^{\alpha_{n}}}, \quad \alpha=\left(\alpha_{1}, \ldots, \alpha_{n}\right), \alpha_{j} \in \mathbb{N}_{0},|\alpha|=\sum_{j=1}^{n}\left|\alpha_{j}\right| .
$$

If $1<p<\infty$ then one has the equivalent characterisation 1.3 1.5 for $W_{p}^{1}\left(\mathbb{R}^{n}\right)$.

Definition 2.1. (i) Let $0<p \leq \infty$ and $s>0$. Let $k \in \mathbb{N}$ with $s \leq k$. Then $\mathbf{L}_{p}^{s}\left(\mathbb{R}^{n}\right)^{k}$ is the collection of all $f \in L_{p}\left(\mathbb{R}^{n}\right)$ for which there exists a function $g \in L_{p}\left(\mathbb{R}^{n}\right)$ with $g(x) \geq 0$ a.e. such that for all $h \in \mathbb{R}^{n}$ with $0<|h| \leq 1$,

$$
|h|^{-s}\left|\Delta_{h}^{k} f(x)\right| \leq \sum_{l=0}^{k} g(x+l h) \quad \text { a.e. in } \mathbb{R}^{n} .
$$

Let

$$
\left\|f\left|\mathbf{L}_{p}^{s}\left(\mathbb{R}^{n}\right)^{k}\|=\| f\right| L_{p}\left(\mathbb{R}^{n}\right)\right\|+\inf \left\|g \mid L_{p}\left(\mathbb{R}^{n}\right)\right\|
$$

where the infimum is taken over all $g$ with 2.4.

(ii) Let $0<p \leq \infty$ and $k \in \mathbb{N}$. Then

$$
\mathbf{W}_{p}^{k}\left(\mathbb{R}^{n}\right)=\mathbf{L}_{p}^{k}\left(\mathbb{R}^{n}\right)^{k} .
$$


REMARK 2.2. Recall that everything must be understood in the context of $\mathbf{M}\left(\mathbb{R}^{n}\right)$, hence in terms of equivalence classes,

$$
\mathbf{L}_{p}^{s}\left(\mathbb{R}^{n}\right)^{k} \hookrightarrow L_{p}\left(\mathbb{R}^{n}\right) \hookrightarrow \mathbf{M}\left(\mathbb{R}^{n}\right) .
$$

This applies in particular to (2.4) for any fixed $h \in \mathbb{R}^{n}, 0<|h| \leq 1$. We add some comments. First we remark that

$$
\mathbf{L}_{p}^{s}\left(\mathbb{R}^{n}\right)^{k} \text { are quasi-Banach spaces. }
$$

If $\left\{f_{j}\right\}$ is a Cauchy sequence in $\mathbf{L}_{p}^{s}\left(\mathbb{R}^{n}\right)^{k}$ then it is also a Cauchy sequence in $L_{p}\left(\mathbb{R}^{n}\right)$. The standard arguments for the completeness of $L_{p}\left(\mathbb{R}^{n}\right)$ as may be found for example in [20, pp. 51-52], must now be complemented by appropriate convergence of optimally chosen functions $g^{j}(x) \geq 0$ according to (2.4), 2.5) with, say, $f_{j+1}-f_{j}$ in place of $f$. Then the same measuretheoretical arguments as in [20] ensure the completeness of $\mathbf{L}_{p}^{s}\left(\mathbb{R}^{n}\right)^{k}$. One may also consult [13, Theorem 5.7, pp. 35-36] for more details. It is sufficient to restrict (1.4) to $|x-y| \leq 1$. Then it follows from the above definition that

$$
\mathbf{W}_{p}^{1}\left(\mathbb{R}^{n}\right)=W_{p}^{1}\left(\mathbb{R}^{n}\right), \quad 1<p<\infty .
$$

This equality (and similar assertions below) have to be understood in the sense that one can find in the equivalence class of each $f \in W_{p}^{1}\left(\mathbb{R}^{n}\right)$ a distinguished representative satisfying 2.4 2.6. Let $k \in \mathbb{N}$. Then $\operatorname{Lip}^{k}\left(\mathbb{R}^{n}\right)$ are Lipschitz spaces collecting all functions $f$ having bounded classical pure derivatives up to order $k-1$ such that

$$
\left\|f\left|\operatorname{Lip}^{k}\left(\mathbb{R}^{n}\right)\|=\| f\right| L_{\infty}\left(\mathbb{R}^{n}\right)\right\|+\sup |h|^{-1}\left|\Delta_{h}^{1} \frac{\partial^{k-1} f}{\partial \nu^{k-1}}(x)\right|<\infty
$$

where the supremum is taken over all directions $\nu \in \mathbb{R}^{n},|\nu|=1$, all $x \in \mathbb{R}^{n}$ and all $h \in \mathbb{R}^{n}$ with $0<|h| \leq 1$. If one replaces in $(2.8)$ the first differences $\Delta_{h}^{1}$ by the second differences $\Delta_{h}^{2}$ then one gets the Zygmund spaces $\mathcal{C}^{k}\left(\mathbb{R}^{n}\right)=$ $B_{\infty, \infty}^{k}\left(\mathbb{R}^{n}\right)$,

$$
\left\|f\left|\mathcal{C}^{k}\left(\mathbb{R}^{n}\right)\|=\| f\right| L_{\infty}\left(\mathbb{R}^{n}\right)\right\|+\sup |h|^{-1}\left|\Delta_{h}^{2} D^{\alpha} f(x)\right|
$$

where the supremum is taken over all $\alpha$ with $|\alpha|=k-1$, all $x \in \mathbb{R}^{n}$ and all $h \in \mathbb{R}^{n}$ with $0<|h| \leq 1$. It is well known that one can replace $D^{\alpha} f$ in 2.9 by $|h|^{-k+1} \Delta_{h}^{k-1} f$. Similarly one has

$$
\left\|f\left|\operatorname{Lip}^{k}\left(\mathbb{R}^{n}\right)\|\sim\| f\right| L_{\infty}\left(\mathbb{R}^{n}\right)\right\|+\sup |h|^{-k}\left|\Delta_{h}^{k} f(x)\right|
$$

where the supremum is taken over all $x \in \mathbb{R}^{n}$ and all $h \in \mathbb{R}^{n}$ with $0<|h| \leq 1$. But in contrast to the corresponding assertions for Zygmund spaces, (2.10) cannot be proved by Fourier-analytical arguments. This formula follows from integral representations of functions, their differences and derivatives, as may be found in [1, Chapter $4, \S 16$, Section 16.1]. Then it follows from 2.4, 2.6 
and well known properties of $\mathcal{C}^{k}\left(\mathbb{R}^{n}\right)$ that

$$
\mathbf{W}_{\infty}^{k}\left(\mathbb{R}^{n}\right)=\operatorname{Lip}^{k}\left(\mathbb{R}^{n}\right) \hookrightarrow \mathcal{C}^{k}\left(\mathbb{R}^{n}\right), \quad \operatorname{Lip}^{k}\left(\mathbb{R}^{n}\right) \neq \mathcal{C}^{k}\left(\mathbb{R}^{n}\right) .
$$

This proves (1.11). If $k=1$ then (1.10) is covered by (2.7). The proof of the corresponding assertion for $2 \leq k \in \mathbb{N}$ is shifted to Section 3. Furthermore one may ask:

$$
\text { Is } \mathbf{L}_{p}^{s}\left(\mathbb{R}^{n}\right)^{k}, 0<p \leq \infty, 0<s<k \in \mathbb{N} \text {, independent of } k \text { ? }
$$

The corresponding assertion for $\mathbf{B}_{p, q}^{s}\left(\mathbb{R}^{n}\right)$ is a cornerstone of the theory of these spaces. One cannot extend the question 2.12) to $s=k$. But (2.4) and also the comparison of $(2.9)$ and $(2.10)$ suggest asking:

$$
\text { Do } \mathbf{L}_{p}^{s}\left(\mathbb{R}^{n}\right)^{k}, 0<p \leq \infty, 0<s<k \in \mathbb{N} \text {, and } \mathbf{B}_{p, \infty}^{s}\left(\mathbb{R}^{n}\right) \text { coincide? }
$$

If $p=\infty$ then by (2.2), 2.3) one has the affirmative answer

$$
\mathbf{L}_{\infty}^{s}\left(\mathbb{R}^{n}\right)^{k}=\mathbf{B}_{\infty, \infty}^{s}\left(\mathbb{R}^{n}\right)=B_{\infty, \infty}^{s}\left(\mathbb{R}^{n}\right)=\mathcal{C}^{s}\left(\mathbb{R}^{n}\right), \quad 0<s<k \in \mathbb{N} .
$$

If $p<\infty$ then there is a second and even more promising candidate. This is the smaller space $\mathbf{F}_{p, \infty}^{s}\left(\mathbb{R}^{n}\right)$. We return to this point in Remark 4.1 below.

2.2. Interpolation and subatomic decompositions. We collect the decisive ingredients to prove the Theorem.

Interpolation. We assume that the reader is familiar with the real interpolation $\left(A_{0}, A_{1}\right)_{\theta, q}$ where $\left\{A_{0}, A_{1}\right\}$ is an interpolation couple of complex quasi-Banach spaces, $0<\theta<1$ and $0<q \leq \infty$. We will specify $A_{0}$ and $A_{1}$ to be $L_{p}\left(\mathbb{R}^{n}\right), 0<p \leq \infty$, and the above spaces $\mathbf{B}_{p, q}^{s}\left(\mathbb{R}^{n}\right)$ and $\mathbf{L}_{p}^{s}\left(\mathbb{R}^{n}\right)^{k}$. All those spaces are continuously embedded in the complete metric space $\mathbf{M}\left(\mathbb{R}^{n}\right)$ mentioned at the beginning of Section 2.1. Hence, real interpolation makes sense. Let $p, q_{1}, q_{2} \in(0, \infty], s>0$, and $0<\theta<1$. Then

$$
\left(L_{p}\left(\mathbb{R}^{n}\right), \mathbf{B}_{p, q_{1}}^{s}\left(\mathbb{R}^{n}\right)\right)_{\theta, q_{2}}=\mathbf{B}_{p, q_{2}}^{s \theta}\left(\mathbb{R}^{n}\right) .
$$

This remarkable interpolation formula is covered by [6. Theorem 6.3, p. 859] where the (bounded) extension domain can be replaced by $\mathbb{R}^{n}$. This theorem has a little history which we discussed in [32, pp. 373-374], where one also finds further related references.

Subatomic decompositions. We follow [34, Sections 9.1, 9.2] based on 33 where one finds further explanations and discussions. We use again standard notation. As usual $\mathbb{Z}^{n}$ stands for the lattice of all points in $\mathbb{R}^{n}$ with integer-valued components. Let $\mathbb{N}_{0}^{n}$ with $n \in \mathbb{N}$ be the set of all multi-indices

$$
\alpha=\left(\alpha_{1}, \ldots, \alpha_{n}\right), \quad \alpha_{j} \in \mathbb{N}_{0}, \quad|\alpha|=\sum_{j=1}^{n}\left|\alpha_{j}\right| .
$$

If $x=\left(x_{1}, \ldots, x_{n}\right) \in \mathbb{R}^{n}$ and $\beta=\left(\beta_{1}, \ldots, \beta_{n}\right) \in \mathbb{N}_{0}^{n}$ then we put

$$
x^{\beta}=x_{1}^{\beta_{1}} \cdots x_{n}^{\beta_{n}} \quad \text { (monomials). }
$$


Let

$$
\mathbb{R}_{++}^{n}=\left\{x \in \mathbb{R}^{n}: x=\left(x_{1}, \ldots, x_{n}\right), x_{j}>0\right\}
$$

and let $K$ be a non-negative $C^{\infty}$ function in $\mathbb{R}^{n}$ with

$$
\operatorname{supp} K \subset\left\{y \in \mathbb{R}^{n}:|y|<2^{J-\varepsilon}\right\} \cap \mathbb{R}_{++}^{n}
$$

for some fixed $\varepsilon>0$ and some fixed natural number $J$ (one may choose $J=n$ once and for all), and

$$
\sum_{m \in \mathbb{Z}^{n}} K(x-m)=1, \quad x \in \mathbb{R}^{n} .
$$

Let

$$
K^{\beta}(x)=\left(2^{-J} x\right)^{\beta} K(x), \quad x \in \mathbb{R}^{n}, \beta \in \mathbb{N}_{0}^{n},
$$

and

$$
K_{j m}^{\beta}(x)=K^{\beta}\left(2^{j} x-m\right), \quad j \in \mathbb{N}_{0}, m \in \mathbb{Z}^{n} .
$$

Obviously, $K_{j m}^{\beta}(x) \geq 0$. We need some sequence spaces. Let $s \in \mathbb{R}$ and $0<p, q \leq \infty$. Then

$$
b_{p, q}^{s}=\left\{\lambda:\left\|\lambda \mid b_{p, q}^{s}\right\|<\infty\right\}
$$

where

$$
\lambda=\left\{\lambda_{j m}^{\beta} \in \mathbb{C}: \beta \in \mathbb{N}_{0}^{n}, j \in \mathbb{N}_{0}, m \in \mathbb{Z}^{n}\right\}
$$

and

$$
\left\|\lambda \mid b_{p, q}^{s}\right\|=\sup _{\beta \in \mathbb{N}_{0}^{n}}\left(\sum_{j=0}^{\infty} 2^{j(s-n / p) q}\left(\sum_{m \in \mathbb{Z}^{n}}\left|\lambda_{j m}^{\beta}\right|^{p}\right)^{q / p}\right)^{1 / q}
$$

with the usual modifications if $p=\infty$ and/or $q=\infty$. Let $\mathbf{B}_{p, q}^{s}\left(\mathbb{R}^{n}\right)$ be the above spaces, quasi-normed by 2.2 . Then $f \in L_{p}\left(\mathbb{R}^{n}\right)$ belongs to $\mathbf{B}_{p, q}^{s}\left(\mathbb{R}^{n}\right)$ if, and only if, it can be represented as

$$
f=\sum_{\beta, j, m} \lambda_{j m}^{\beta} K_{j m}^{\beta}, \quad \lambda \in b_{p, q}^{s} .
$$

Furthermore,

$$
\left\|f\left|\mathbf{B}_{p, q}^{s}\left(\mathbb{R}^{n}\right)\|\sim \inf \| \lambda\right| b_{p, q}^{s}\right\|
$$

where the infimum is taken over all admissible representations (2.21).

We add a few comments. If $p<\infty$ then the series in 2.21) converges absolutely in $L_{p}\left(\mathbb{R}^{n}\right)$ and hence in $\mathbf{M}\left(\mathbb{R}^{n}\right)$. If $p=\infty$ then 2.21) converges absolutely in the weighted space $L_{\infty}\left(\mathbb{R}^{n}, w_{\sigma}\right), \sigma>0$, normed by

$$
\left\|g\left|L_{\infty}\left(\mathbb{R}^{n}, w_{\sigma}\right)\|=\| w_{\sigma} f\right| L_{\infty}\left(\mathbb{R}^{n}\right)\right\|, \quad w_{\sigma}(x)=\left(1+|x|^{2}\right)^{\sigma / 2},
$$

and hence again in $\mathbf{M}\left(\mathbb{R}^{n}\right)$. The representation (2.21), 2.22) is neither a basis nor a frame. The above assertion is covered by [34, Definitions 9.6, 
9.12, Proposition 9.14, pp. 382, 387-389]. The proofs in [33, 34 rely in a decisive way on atomic decompositions of $\mathbf{B}_{p, q}^{s}\left(\mathbb{R}^{n}\right)$ due to Yu. V. Netrusov [22] and Hedberg and Netrusov [12].

3. Proof of the Theorem. In this section we prove the Theorem as stated in the Introduction.

Step 1. Let $f \in \mathbf{L}_{p}^{s}\left(\mathbb{R}^{n}\right)^{k}$ according to Definition 2.1. Let $0<s \leq k<M$ $\in \mathbb{N}$ and $h \in \mathbb{R}^{n}, 0<|h| \leq t<1$. If $g$ in 2.4), 2.5) is optimally chosen then

$$
\left\|\Delta_{h}^{M} f\left|L_{p}\left(\mathbb{R}^{n}\right)\left\|\leq c t^{s}\right\| f\right| \mathbf{L}_{p}^{s}\left(\mathbb{R}^{n}\right)^{k}\right\| .
$$

Then it follows from $(2.2)$ that

$$
\begin{aligned}
\left\|f \mid \mathbf{B}_{p, \infty}^{s}\left(\mathbb{R}^{n}\right)\right\|_{M} & =\left\|f\left|L_{p}\left(\mathbb{R}^{n}\right)\left\|+\sup _{0<|h| \leq t \leq 1} t^{-s}\right\| \Delta_{h}^{M} f\right| L_{p}\left(\mathbb{R}^{n}\right)\right\| \\
& \leq c\left\|f \mid \mathbf{L}_{p}^{s}\left(\mathbb{R}^{n}\right)^{k}\right\| .
\end{aligned}
$$

This proves the right-hand embedding of (1.8).

Step 2. We prove the left-hand embedding of $(1.8)$. We represent $f \in$ $\mathbf{B}_{p, q}^{s}\left(\mathbb{R}^{n}\right)$ with $0<p \leq \infty, 0<q \leq 1$, by 2.21, 2.22 based on 2.16 (2.19). Let $\chi_{j m}$ be the characteristic function of the ball

$$
\left\{x \in \mathbb{R}^{n}:\left|x-2^{-j} m\right|<2^{J-j}\right\}, \quad j \in \mathbb{N}_{0}, m \in \mathbb{Z}^{n} .
$$

Then it follows from (2.16) and (2.18), 2.19) that for any $\gamma \in \mathbb{N}_{0}^{n}$ there is a constant $c_{\gamma}>0$ such that

$$
\left|D^{\gamma} K_{j m}^{\beta}(x)\right| \leq c_{\gamma} 2^{j|\gamma|} 2^{-\varepsilon|\beta|} \chi_{j m}(x), \quad x \in \mathbb{R}^{n},
$$

for all $\beta \in \mathbb{N}_{0}^{n}, j \in \mathbb{N}_{0}, m \in \mathbb{Z}^{n}$. Let $0<s \leq k \in \mathbb{N}$. By (2.21) we have, for $0<|h| \leq 1, h \in \mathbb{R}^{n}$,

$$
|h|^{-s} \Delta_{h}^{k} f(x)=\sum_{\beta, j, m} \lambda_{j m}^{\beta}|h|^{-s} \Delta_{h}^{k} K_{j m}^{\beta}(x) .
$$

If $s<k$ then it follows from the homogeneity property of $\mathcal{C}^{s}\left(\mathbb{R}^{n}\right)=B_{\infty, \infty}^{s}\left(\mathbb{R}^{n}\right)$ according to [35, Theorem 2.11, p. 34] that for $x \in \mathbb{R}^{n}, \beta \in \mathbb{N}_{0}^{n}, j \in \mathbb{N}_{0}$, $m \in \mathbb{Z}^{n}$,

$$
|h|^{-s}\left|\Delta_{h}^{k} K_{j m}^{\beta}(x)\right| \leq c 2^{-\varepsilon|\beta|} 2^{j s} \sum_{l=0}^{k} \chi_{j m}(x+l h) .
$$

But this can also be proved directly. If $s=k$ then it follows from (2.10), (3.1) and well-known assertions that

$$
|h|^{-k}\left|\Delta_{h}^{k} K_{j m}^{\beta}(x)\right| \leq c\left\|K_{j m}^{\beta}\left|\operatorname{Lip}^{k}\left(\mathbb{R}^{n}\right)\left\|\leq c^{\prime} \sum_{|\alpha|=k}\right\| D^{\alpha} K_{j m}^{\beta}\right| L_{\infty}\left(\mathbb{R}^{n}\right)\right\|
$$


and hence for $x \in \mathbb{R}^{n}, \beta \in \mathbb{N}_{0}^{n}, j \in \mathbb{N}_{0}, m \in \mathbb{Z}^{n}$,

$$
|h|^{-k}\left|\Delta_{h}^{k} K_{j m}^{\beta}(x)\right| \leq c 2^{-\varepsilon|\beta|} 2^{j k} \sum_{l=0}^{k} \chi_{j m}(x+l h) .
$$

Inserted in 3.2 one has in all cases

$$
|h|^{-s}\left|\Delta_{h}^{k} f(x)\right| \leq \sum_{l=0}^{k} g(x+l h), \quad x \in \mathbb{R}^{n},
$$

with

$$
g(x)=c \sum_{\beta \in \mathbb{N}_{0}^{n}} 2^{-\varepsilon|\beta|} \sum_{j=0}^{\infty} 2^{j s} \sum_{m \in \mathbb{Z}^{n}}\left|\lambda_{j m}^{\beta}\right| \chi_{j m}(x), \quad \lambda \in b_{p, q}^{s} .
$$

If $p \geq 1$ then

$$
\begin{aligned}
\left\|g \mid L_{p}\left(\mathbb{R}^{n}\right)\right\| & \leq c \sum_{\beta \in \mathbb{N}_{0}^{n}} 2^{-\varepsilon|\beta|} \sum_{j=0}^{\infty} 2^{j s-j n / p}\left(\sum_{m \in \mathbb{Z}^{n}}\left|\lambda_{j m}^{\beta}\right|^{p}\right)^{1 / p} \\
& \leq c\left\|\lambda\left|b_{p, 1}^{s}\left\|\leq c^{\prime}\right\| f\right| \mathbf{B}_{p, 1}^{s}\left(\mathbb{R}^{n}\right)\right\|,
\end{aligned}
$$

where we assume that $\lambda$ in 2.20$), 2.22$ is optimally chosen. If $0<p<1$ then we apply the $p$-triangle inequality to 3.5 and obtain

$$
\left\|g\left|L_{p}\left(\mathbb{R}^{n}\right)\left\|^{p} \leq c\right\| \lambda\right| b_{p, p}^{s}\right\|^{p} \leq c^{\prime}\left\|f \mid \mathbf{B}_{p, p}^{s}\left(\mathbb{R}^{n}\right)\right\|^{p} .
$$

Now the left-hand embedding of 1.8 follows from (3.6), 3.7) and Definition 2.1.

Step 3. The interpolation (1.9) follows from (1.8), 2.15) and the reiteration theorem of interpolation theory.

Step 4. We prove part (iii), where (1.11) is covered by (2.11). If $1<p<$ $\infty$ and $k=1$ then 1.10 coincides with (2.7). Let $B(x, r)$ be a ball in $\mathbb{R}^{n}$, centred at $x \in \mathbb{R}^{n}$ and of radius $r>0$. Then

$$
\left(M^{1} f\right)(x)=(M f)(x)=\sup _{r>0} r^{-n} \int_{B(x, r)}|f(y)| d y
$$

is the usual Hardy-Littlewood maximal function (up to a constant). According to [8] there is a constant $c>0$ such that for all $f \in W_{p}^{1}\left(\mathbb{R}^{n}\right), x \in \mathbb{R}^{n}$ and $h \in \mathbb{R}^{n}$ with $0<|h| \leq 1$,

$$
|h|^{-1}\left|\Delta_{h}^{1} f(x)\right| \leq c \sum_{|\alpha|=1}\left[M\left(D^{\alpha} f\right)(x)+M\left(D^{\alpha} f\right)(x+h)\right] .
$$

Let

$$
\left(M^{k} f\right)(x)=M\left(M^{k-1} f\right)(x), \quad 2 \leq k \in \mathbb{N},
$$


be the iteration of $M$ in 3.8 . Then it follows from 3.9 that

$$
|h|^{-k}\left|\Delta_{h}^{k} f(x)\right| \leq c \sum_{l=0}^{k} \sum_{|\alpha|=k} M^{k}\left(D^{\alpha} f\right)(x+l h)
$$

for some $c>0$, and all $f \in W_{p}^{k}\left(\mathbb{R}^{n}\right), x \in \mathbb{R}^{n}, h \in \mathbb{R}^{n}$ with $0<|h| \leq 1$. Since $M^{k}$ is a bounded map in $L_{p}\left(\mathbb{R}^{n}\right)$ with $1<p<\infty$ it follows from Definition 2.1 that $f \in \mathbf{W}_{p}^{k}\left(\mathbb{R}^{n}\right)$ and

$$
\left\|f\left|\mathbf{W}_{p}^{k}\left(\mathbb{R}^{n}\right)\|\leq c\| f\right| W_{p}^{k}\left(\mathbb{R}^{n}\right)\right\|, \quad f \in W_{p}^{k}\left(\mathbb{R}^{n}\right) .
$$

We prove the converse and assume that $f \in \mathbf{W}_{p}^{k}\left(\mathbb{R}^{n}\right)$. Let $g \in L_{p}\left(\mathbb{R}^{n}\right)$ be a corresponding dominating function according to (2.4) with $s=k$. For $\varphi \in S\left(\mathbb{R}^{n}\right)$ one has

$$
\int_{\mathbb{R}^{n}}|h|^{-k} \Delta_{h}^{k} f(x) \varphi(x) d x=\int_{\mathbb{R}^{n}} f(x)|h|^{-k} \Delta_{-h}^{k} \varphi(x) d x .
$$

Let $h=\left(h_{1}, 0, \ldots, 0\right)$ with $h_{1}>0$. Then

$$
\lim _{h_{1} \rightarrow 0} h_{1}^{-k} \Delta_{-h}^{k} \varphi(x)=(-1)^{k} \frac{\partial^{k} \varphi}{\partial x_{1}^{k}}(x)
$$

uniformly in $x \in \mathbb{R}^{n}$. To justify 3.12 , we may assume that the Fourier transform $F \varphi$ of $\varphi$ has a compact support. Then

$F\left[h_{1}^{-k} \Delta_{-h}^{k} \varphi-(-1)^{k} \frac{\partial^{k} \varphi}{\partial x_{1}^{k}}\right](\xi)=\left[h_{1}^{-k}\left(e^{-i h_{1} \xi_{1}}-1\right)^{k}-\left(-i \xi_{1}\right)^{k}\right] F \varphi(\xi), \quad \xi \in \mathbb{R}^{n}$, converges in $S\left(\mathbb{R}^{n}\right)$ to zero. Then one obtains $(3.12)$ in $S\left(\mathbb{R}^{n}\right)$ and the corresponding convergence on the right-hand side of (3.11). For the left-hand side of (3.11), we remark that $|h|^{-k} \Delta_{h}^{k} f(x)$ again with $h=\left(h_{1}, 0, \ldots, 0\right), h_{1}>0$, is a bounded set in the reflexive spaces $L_{p}\left(\mathbb{R}^{n}\right)$, and hence weakly precompact. Let $f^{k}$ be the limit of a corresponding weakly convergent sequence. Then it follows that

$$
\int_{\mathbb{R}^{n}} f^{k}(x) \varphi(x) d x=(-1)^{k} \int_{\mathbb{R}^{n}} f(x) \frac{\partial^{k} \varphi}{\partial x_{1}^{k}}(x) d x
$$

and

$$
\left\|f^{k}\left|L_{p}\left(\mathbb{R}^{n}\right)\|\leq c\| g\right| L_{p}\left(\mathbb{R}^{n}\right)\right\| .
$$

This proves $f^{k}=\partial^{k} f / \partial x_{1}^{k} \in L_{p}\left(\mathbb{R}^{n}\right)$ distributionally and

$$
f \in W_{p}^{k}\left(\mathbb{R}^{n}\right) \quad \text { with } \quad\left\|f\left|W_{p}^{k}\left(\mathbb{R}^{n}\right)\|\leq c\| f\right| \mathbf{W}_{p}^{k}\left(\mathbb{R}^{n}\right)\right\| .
$$

Together with (3.10) one obtains (1.10). This type of argument is not new. It goes back to S. L. Sobolev [29, §5.2, pp. 41-43] and has been used afterwards by several authors including [23, p. 177]. 
4. Complements. We add a few comments and further references complementing the above considerations.

REMARK 4.1. First we return to Remark 2.2. If $p=\infty$ then one has (2.11), 2.14). Otherwise there is the classical observation 2.7 by B. Bojarski and P. Hajłasz complemented now by (1.10). According to [17 one can extend (2.7), based on (the inhomogeneous version of) 1.4 , 1.5 to all distributionally defined first-order Sobolev (-Hardy) spaces which can be continuously embedded into some $L_{r}\left(\mathbb{R}^{n}\right)$ with $r>1$. In other words,

$$
\mathbf{W}_{p}^{1}\left(\mathbb{R}^{n}\right)=H_{p}^{1}\left(\mathbb{R}^{n}\right)=F_{p, 2}^{1}\left(\mathbb{R}^{n}\right), \quad \frac{n}{n+1}<p<\infty .
$$

Recall that $H_{p}^{1}\left(\mathbb{R}^{n}\right)=W_{p}^{1}\left(\mathbb{R}^{n}\right)$ if $1<p<\infty$ whereas the Hardy-Sobolev space $H_{1}^{1}\left(\mathbb{R}^{n}\right)$ is smaller than the Sobolev space $W_{1}^{1}\left(\mathbb{R}^{n}\right)$ normed by 1.2 ) with $p=1$. What can be said about $W_{1}^{1}\left(\mathbb{R}^{n}\right)$ in the above context has been studied in detail in [9]. If $p=n /(n+1)$ then $H_{n /(n+1)}^{1}\left(\mathbb{R}^{n}\right)$ is still continuously embedded in $L_{1}\left(\mathbb{R}^{n}\right)$ and it makes sense to ask whether 4.1) can be extended to this case. But this is unlikely. In this limiting situation the diverse settings of $B$-spaces and $F$-spaces behave rather differently as came out quite recently, [26, 27]. We complemented the above assertions by the question (2.13). The right-hand side of the inclusion 1.8 and also the question 2.13) can be strengthened as follows. Let $\Delta_{h}^{k} f(x)$ be the differences (2.1) and let $f \in L_{p}\left(\mathbb{R}^{n}\right)$ with $0<p<\infty$. Then

$$
d_{t, p}^{k} f(x)=\left(t^{-n} \int_{|h| \leq t}\left|\left(\Delta_{h}^{k} f\right)(x)\right|^{p} d h\right)^{1 / p}, \quad 0<t<\infty, x \in \mathbb{R}^{n},
$$

are local ball means. Let $0<p<\infty, 0<q \leq \infty, 0<s<k \in \mathbb{N}$. Then $\mathbf{F}_{p, q}^{s}\left(\mathbb{R}^{n}\right)$ is the collection of all $f \in L_{p}\left(\mathbb{R}^{n}\right)$ such that

$$
\left\|f\left|\mathbf{F}_{p, q}^{s}\left(\mathbb{R}^{n}\right)\left\|_{k}=\right\| f\right| L_{p}\left(\mathbb{R}^{n}\right)\right\|+\left\|\left(\int_{0}^{1} t^{-s q} d_{t, p}^{k} f(\cdot)^{q} \frac{d t}{t}\right)^{1 / q} \mid L_{p}\left(\mathbb{R}^{n}\right)\right\|
$$

is finite, where

$$
\left\|f\left|\mathbf{F}_{p, \infty}^{s}\left(\mathbb{R}^{n}\right)\left\|_{k}=\right\| f\right| L_{p}\left(\mathbb{R}^{n}\right)\right\|+\left\|\sup _{0<t<1} t^{-s} d_{t, p}^{k} f(\cdot) \mid L_{p}\left(\mathbb{R}^{n}\right)\right\|
$$

is the usual modification if $q=\infty$.

We refer to [34, Chapter 9], based on [33], where we developed the theory of these spaces parallel to the spaces $\mathbf{B}_{p, q}^{s}\left(\mathbb{R}^{n}\right)$. Compared with the better known Fourier-analytically defined spaces $F_{p, q}^{s}\left(\mathbb{R}^{n}\right)$ one has

$$
\begin{aligned}
& \mathbf{F}_{p, q}^{s}\left(\mathbb{R}^{n}\right)=F_{p, q}^{s}\left(\mathbb{R}^{n}\right) \\
& \text { if } 0<p<\infty, 0<q \leq \infty, s>n\left(\frac{1}{\min (p, q)}-\frac{1}{\max (1, p)}\right),
\end{aligned}
$$


as a counterpart of (2.3). Furthermore,

$$
\mathbf{B}_{p, \min (p, q)}^{s}\left(\mathbb{R}^{n}\right) \hookrightarrow \mathbf{F}_{p, q}^{s}\left(\mathbb{R}^{n}\right) \hookrightarrow \mathbf{B}_{p, \max (p, q)}^{s}\left(\mathbb{R}^{n}\right)
$$

for $s>0,0<p<\infty, 0<q \leq \infty$. We refer to [36. Section 1.1.8] and the recent papers [11, 25]. Of interest for us are the spaces $\mathbf{F}_{p, \infty}^{s}\left(\mathbb{R}^{n}\right)$. Then one has

$$
\mathbf{F}_{p, \infty}^{s}\left(\mathbb{R}^{n}\right)=F_{p, \infty}^{s}\left(\mathbb{R}^{n}\right) \quad \text { if } s>n \max (0,1 / p-1)
$$

as in 2.3. The right-hand embedding of 1.8 can be strengthened to

$$
\mathbf{L}_{p}^{s}\left(\mathbb{R}^{n}\right)^{k} \hookrightarrow \mathbf{F}_{p, \infty}^{s}\left(\mathbb{R}^{n}\right), \quad 0<p<\infty, 0<s<k \in \mathbb{N} .
$$

For the proof we first remark that one can replace $d_{t, p}^{k} f$ in 4.3), 4.4 by $d_{t, u}^{k} f$ with $0<u \leq p$. This is covered for the spaces $F_{p, q}^{s}\left(\mathbb{R}^{n}\right)$ in 4.5, 4.6 by [31, Theorem 3.5.3, p. 194]. The extension to all spaces $\mathbf{F}_{p, q}^{s}\left(\mathbb{R}^{n}\right)$ is not explicitly mentioned in [34 but is a consequence of the underlying assertion [12, Theorem 1.1.14, p. 14]. Let $f \in \mathbf{L}_{p}^{s}\left(\mathbb{R}^{n}\right)^{k}$. We insert 2.4 in 4.2 with $d_{t, u}^{k} f, u<p$, to obtain

$$
d_{t, u}^{k} f(x) \leq c t^{s}\left(M|g|^{u}\right)^{1 / u}(x), \quad 0<t \leq 1, x \in \mathbb{R}^{n},
$$

where $M$ is the usual Hardy-Littlewood maximal function. Then it follows from (4.4) with $d_{t, u}^{k} f$ in place of $d_{t, p}^{k} f$ that

$$
\left\|f\left|\mathbf{F}_{p, \infty}^{s}\left(\mathbb{R}^{n}\right)\|\leq c\|\left(M|g|^{u}\right)^{1 / u}\right| L_{p}\left(\mathbb{R}^{n}\right)\right\| \leq c^{\prime}\left\|g \mid L_{p}\left(\mathbb{R}^{n}\right)\right\| .
$$

This proves (4.7). Now one can replace 2.13 by the sharper question:

Do $\mathbf{L}_{p}^{s}\left(\mathbb{R}^{n}\right)^{k}, 0<p<\infty, 0<s<k \in \mathbb{N}$, and $\mathbf{F}_{p, \infty}^{s}\left(\mathbb{R}^{n}\right)$ coincide?

There are a few affirmative answers in the context of the spaces $F_{p, \infty}^{s}\left(\mathbb{R}^{n}\right)$ (and their extensions to some metric spaces $X$ ). Dachun Yang proved in [38, Corollary 1.3, p. 686] that

$$
\mathbf{L}_{p}^{s}\left(\mathbb{R}^{n}\right)^{1}=F_{p, \infty}^{s}\left(\mathbb{R}^{n}\right), \quad 1<p<\infty, 0<s<1 .
$$

This has been extended substantially in [18] dealing with counterparts of 4.1). This leads in particular to assertions of type (4.8) with $0<s<1$, $n /(n+s)<p<\infty$. Again it makes sense to ask what happens in the limiting case $p=n /(s+n)$. But as said above it is unlikely that this delicate case can be incorporated in this theory. Furthermore we refer to [21] for recent characterisations of spaces of type $F_{p, q}^{s}(X)$ on some metric spaces in terms of first differences. This fits in the above scheme at least if $X=\mathbb{R}^{n}$.

REMARK 4.2. Sharp Sobolev embeddings between the distributionally defined spaces $B_{p, q}^{s}\left(\mathbb{R}^{n}\right), F_{p, q}^{s}\left(\mathbb{R}^{n}\right)$ (and their special cases) are a cornerstone of the related theory of function spaces and its applications. We recall two outstanding assertions. Let

$$
1<p<\infty, \quad 0<\sigma=n / r-n / p .
$$


Then

$$
B_{r, q}^{\sigma}\left(\mathbb{R}^{n}\right) \hookrightarrow L_{p}\left(\mathbb{R}^{n}\right) \text { if, and only if, } \quad 0<q \leq p .
$$

Furthermore,

$$
F_{r, q}^{\sigma}\left(\mathbb{R}^{n}\right) \hookrightarrow F_{r, \infty}^{\sigma}\left(\mathbb{R}^{n}\right) \hookrightarrow L_{p}\left(\mathbb{R}^{n}\right) \quad \text { for all } 0<q \leq \infty .
$$

One may consult [32, Theorem 11.4, p. 170] and the references given there, in particular [28]. Let $H_{p}^{s}\left(\mathbb{R}^{n}\right)=F_{p, 2}^{s}\left(\mathbb{R}^{n}\right)$ be the usual (fractional) Sobolev spaces. Let $p, r, \sigma$ be as in 4.9 and let $s \in \mathbb{R}$. Then it follows from 4.10), 4.11 by lifting that

$$
B_{r, q}^{\sigma+s}\left(\mathbb{R}^{n}\right) \hookrightarrow H_{p}^{s}\left(\mathbb{R}^{n}\right) \text { if, and only if, } \quad 0<q \leq p,
$$

and

$$
F_{r, q}^{\sigma+s}\left(\mathbb{R}^{n}\right) \hookrightarrow F_{r, \infty}^{\sigma+s}\left(\mathbb{R}^{n}\right) \hookrightarrow H_{p}^{s}\left(\mathbb{R}^{n}\right) \quad \text { for all } 0<q \leq \infty .
$$

We ask for counterparts of 4.10, 4.11 and also of 4.12, 4.13 for Bspaces, F-spaces and with $\mathbf{L}_{p}^{s}\left(\mathbb{R}^{n}\right)^{k}$ in place of $H_{p}^{s}\left(\mathbb{R}^{n}\right)$.

Let

$$
0<p<\infty, \quad 0<\sigma=n / r-n / p \text {. }
$$

Then

$$
\mathbf{B}_{r, q}^{\sigma}\left(\mathbb{R}^{n}\right) \hookrightarrow \mathbf{B}_{r, p}^{\sigma}\left(\mathbb{R}^{n}\right) \hookrightarrow L_{p}\left(\mathbb{R}^{n}\right) \quad \text { if } 0<q \leq p,
$$

[11, Theorem 1.15, (1.34), pp. 732-733], and

$$
\mathbf{F}_{r, q}^{\sigma}\left(\mathbb{R}^{n}\right) \hookrightarrow \mathbf{F}_{r, \infty}^{\sigma}\left(\mathbb{R}^{n}\right) \hookrightarrow L_{p}\left(\mathbb{R}^{n}\right) \quad \text { for all } 0<q \leq \infty,
$$

[25. Proposition 2.19, pp. 267-268].

Based on these observations we prove the following counterparts of 4.12, 4.13.

$$
0<p<\infty, \quad 0<s \leq k \in \mathbb{N}, \quad 0<\sigma=n / r-n / p
$$

Then

$$
\mathbf{B}_{r, q}^{s+\sigma}\left(\mathbb{R}^{n}\right) \hookrightarrow \mathbf{L}_{p}^{s}\left(\mathbb{R}^{n}\right)^{k} \quad \text { if } 0<q \leq p
$$

and

$$
\mathbf{F}_{r, q}^{s+\sigma}\left(\mathbb{R}^{n}\right) \hookrightarrow \mathbf{F}_{r, \infty}^{s+\sigma}\left(\mathbb{R}^{n}\right) \hookrightarrow \mathbf{L}_{p}^{s}\left(\mathbb{R}^{n}\right)^{k} \quad \text { for all } 0<q \leq \infty .
$$

For the proof of these assertions we first remark that one can replace the characteristic functions $\chi_{j m}$ in 3.3 by

$$
0 \leq \psi_{j m} \in C^{\infty}\left(\mathbb{R}^{n}\right), \quad \operatorname{supp} \psi_{j m} \subset\left\{x \in \mathbb{R}^{n}:\left|x-2^{-j} m\right|<c 2^{J-j}\right\}
$$

with

$$
\psi_{j m}(x)=1 \quad \text { if }\left|x-2^{-j} m\right| \leq 2^{J-\varepsilon-j}
$$


One may think about

$$
\psi_{j m}(x)=\sum_{|l-m| \leq a} K_{j l}^{0}(x),
$$

according to 2.19) and with a suitably chosen $a>0$. Then one has again (3.4), now with

$$
g(x)=c \sum_{\beta \in \mathbb{N}_{0}^{n}} 2^{-\varepsilon|\beta|} \sum_{j=0}^{\infty} 2^{j s} \sum_{m \in \mathbb{Z}^{n}}\left|\lambda_{j m}^{\beta}\right| \psi_{j m}(x), \quad \lambda \in b_{r, q}^{s+\sigma},
$$

in place of (3.5). We interpret (4.18) as a subatomic decomposition in $\mathbf{B}_{r, q}^{\sigma}\left(\mathbb{R}^{n}\right)$. Then it follows from $2.20-2.22$ and an optimal choice of $\lambda$ that

$$
\begin{aligned}
\left\|g \mid \mathbf{B}_{r, q}^{\sigma}\left(\mathbb{R}^{n}\right)\right\| & \leq c \sup _{\beta \in \mathbb{N}_{0}^{n}}\left(\sum_{j=0}^{\infty} 2^{j(s+\sigma-n / r) q}\left(\sum_{m \in \mathbb{Z}^{n}}\left|\lambda_{j m}^{\beta}\right|^{r}\right)^{q / r}\right)^{1 / q} \\
& \leq c^{\prime}\left\|f \mid \mathbf{B}_{r, q}^{s+\sigma}\left(\mathbb{R}^{n}\right)\right\| .
\end{aligned}
$$

Together with (4.14) one obtains the counterpart of (3.6) and hence (4.16). Then (4.17) follows from 4.15) and the corresponding subatomic decompositions for $\mathbf{F}_{r, \infty}^{s+\sigma}\left(\mathbb{R}^{n}\right)$ according to [34, Definition 9.4, Proposition 9.14, pp. 381, 388].

At first glance the situation looks a little bit curious. On one hand one has the rather final sharp Sobolev embeddings (4.16), 4.17). On the other hand, the left-hand embedding of $(1.8)$ is less satisfactory (but sufficient to justify (1.9p). The difference comes from (4.18). It can be interpreted as a subatomic decomposition in $\mathbf{B}_{r, q}^{\sigma}\left(\mathbb{R}^{n}\right)$, but not in $L_{p}\left(\mathbb{R}^{n}\right)$. The corresponding estimate in (3.6) is obtained by brute force. One may ask whether expansions of type (4.18) can be interpreted as atomic decompositions in, say, $L_{p}\left(\mathbb{R}^{n}\right)=F_{p, 2}^{0}\left(\mathbb{R}^{n}\right), 1<p<\infty$, in the context of distributionally defined spaces on $\mathbb{R}^{n}$. It was an open problem for a long time whether one really needs first moment conditions for atomic expansions of the spaces $B_{p, q}^{0}\left(\mathbb{R}^{n}\right)$ and $F_{p, q}^{0}\left(\mathbb{R}^{n}\right)$, say, with $1<p, q<\infty$, including $L_{p}\left(\mathbb{R}^{n}\right)$ and in particular $L_{2}\left(\mathbb{R}^{n}\right)$. It came out only quite recently that first moment conditions are indispensable. We refer to [37, Remark 3.7, pp. 32-33], extended afterwards in [26, Section 3.2, pp. 126-127] and [27, Section 3.2, pp. 160-161].

REMARK 4.3. We return to Remark 4.1. With respect to the Fourieranalytically defined spaces one has so far the identification (4.1) extending Hajłasz's observation 2.7) to the Hardy-Sobolev spaces $F_{p, 2}^{1}\left(\mathbb{R}^{n}\right)=H_{p}^{1}\left(\mathbb{R}^{n}\right)$, $n /(n+1)<p \leq 1$. If $0<s<1$ then we mentioned above that Dachun Yang's assertion 4.8) has been extended in [18] to $n /(n+s)<p<\infty$. One 
may ask whether

$$
\begin{cases}F_{p, 2}^{1}\left(\mathbb{R}^{n}\right), & n /(n+1)<p<\infty \\ F_{p, \infty}^{s}\left(\mathbb{R}^{n}\right), & 0<s<1, n /(n+s)<p<\infty,\end{cases}
$$

are exceptional spaces admitting pointwise characterisations of the above type. But according to the recent paper [19] (almost) all (homogeneous) spaces of type $B_{p, q}^{s}\left(\mathbb{R}^{n}\right)$ and $F_{p, q}^{s}\left(\mathbb{R}^{n}\right)$ with $0<s<1$ and $n /(n+s)<p<\infty$ can be described by appropriate modifications of $(2.4)$ with $k=1$ (first differences). One has to replace $g$ by $g_{j}$ if $2^{-j} \leq|h|<2^{-j+1}$ and $g \in L_{p}\left(\mathbb{R}^{n}\right)$ in 2.5 by $\left\{g_{j}\right\}_{j=0}^{\infty} \in \ell_{q}\left(L_{p}\left(\mathbb{R}^{n}\right)\right)$ for $B$-spaces and $\left\{g_{j}\right\}_{j=0}^{\infty} \in L_{p}\left(\mathbb{R}^{n}, \ell_{q}\right)$ for $F$-spaces. Adapted to our situation, a corresponding modification of Definition 2.1 may look as follows. One replaces 2.4 by

$$
|h|^{-s}\left|\Delta_{h}^{k} f(x)\right| \leq \sum_{l=0}^{k} g_{j}(x+l h), \quad 2^{-j} \leq|h|<2^{-j+1},
$$

where $j \in \mathbb{N}$, and $\left\|g \mid L_{p}\left(\mathbb{R}^{n}\right)\right\|$ in 2.5 by

$$
\begin{cases}\left(\sum_{j=1}^{\infty}\left\|g_{j} \mid L_{p}\left(\mathbb{R}^{n}\right)\right\|^{q}\right)^{1 / q} & \text { in the case of } \mathbf{B} \text {-spaces } \\ \left\|\left(\sum_{j=1}^{\infty}\left|g_{j}(x)\right|^{q}\right)^{1 / q} \mid L_{p}\left(\mathbb{R}^{n}\right)\right\| & \text { in the case of } \mathbf{F} \text {-spaces. }\end{cases}
$$

The question arises what can be said about the corresponding spaces introduced in this way and whether they coincide with $\mathbf{B}_{p, q}^{s}\left(\mathbb{R}^{n}\right)$ and $\mathbf{F}_{p, q}^{s}\left(\mathbb{R}^{n}\right)$.

REMARK 4.4. So far we dealt exclusively with spaces on $\mathbb{R}^{n}$. There is little doubt that these considerations can be extended to, say, bounded Lipschitz domains $\Omega$ in $\mathbb{R}^{n}$. In particular 2.15 remains valid if one replaces $\mathbb{R}^{n}$ by bounded Lipschitz domains (with the same references as there). On the other hand the observation $\sqrt{1.4}, 1.5$ was just the point of departure in 8 to introduce and study first order Sobolev spaces $W_{p}^{1}(X, \varrho, \mu), 1 \leq p \leq \infty$, on, say, metric spaces $(X, \varrho)$ furnished with a doubling Radon measure $\mu$. Nowadays there are several proposals for first order Sobolev spaces and related Besov spaces of type $B_{p, q}^{s}$ with $0<s<1,1 \leq p \leq \infty, 1 \leq q \leq \infty$. This theory becomes especially rich and coherent if one assumes in addition that $(X, \varrho, \mu)$ supports some Poincaré inequalities. We refer again to [7, 8, 21, 38 and in particular to [10, 16. One may ask whether spaces $\mathbf{L}_{p}^{s}(X, \varrho, \mu)^{k}$ similar to those in Definition 2.1 make sense. This seems to be the case if $0<p \leq \infty$ and $0<s \leq 1=k \in \mathbb{N}$ (first differences). The subatomic decompositions in Section 2.2 have a counterpart for spaces on sets ([34, Section 9.3], based on [33]). Even the extension of this theory to spaces defined by higher differences $\Delta_{h}^{k}$ with $2 \leq k \in \mathbb{N}$ is not so hopeless as it seems to be at first glance. First one can deal with the metric space $\left(\mathbb{R}^{n}, \varrho_{L}^{\varepsilon}, \mu_{L}\right)$, where $\varrho_{L}$ is the Euclidean distance, $0<\varepsilon<1$, and the Lebesgue measure $\mu_{L}$. Here $\mathbb{R}^{n}$ can be replaced by the unit cube in $\mathbb{R}^{n}$ or 
the unit interval $I=(0,1)$ in case of $n=1$. Afterwards the snowflaked bi-Lipschitzian transform maps these metric spaces onto distinguished fractals in higher-dimensional Euclidean spaces. One may consult [34, Sections 1.17.6, 8.2, pp. 119-120] for details and references. The most prominent example is the Koch curve (or snowflake) in $\mathbb{R}^{2}$ furnished with the Euclidean distance in $\mathbb{R}^{2}$ and the Hausdorff measure $\mathcal{H}^{d}, d=\log 4 / \log 3$, as the biLipschitzian image of the unit interval $I=(0,1)$, furnished with the metric $\varrho_{L}^{1 / d}$ and the Lebesgue measure. This may pave the way to transferring the above theory to some distinguished fractals, to defining higher differences on these fractals and to comparing the outcome with already existing function spaces on these sets. First steps in this direction have been made in 14, 15.

Acknowledgements. I wish to thank the referee for careful reading and valuable comments.

\section{References}

[1] O. V. Besov, V. P. Il'in and S. M. Nikol'skiı̌, Integral Representations of Functions and Imbedding Theorems, Nauka, Moscow, 1975, 2nd ed. 1996 (in Russian); English transl.: Wiley, New York, 1978/79.

[2] B. Bojarski, Pointwise characterization of Sobolev classes, Proc. Steklov Inst. Math. 255 (2006), 65-81.

[3] —, Whitney's jets for Sobolev functions, Ukrainian Math. J. 59 (2007), 379-395.

[4] B. Bojarski and P. Hajłasz, Pointwise inequalities for Sobolev functions and some applications, Studia Math. 106 (1993), 77-92.

[5] B. Bojarski, P. Hajłasz and P. Strzelecki, Improved $C^{k, \lambda}$ approximation of higher order Sobolev functions in norm and capacity, Indiana Univ. Math. J. 51 (2002), 507-540.

[6] R. A. DeVore and R. Sharpley, Besov spaces on domains in $\mathbb{R}^{d}$, Trans. Amer. Math. Soc. 335 (1993), 843-864.

[7] A. Gogatishvili, P. Koskela and N. Shanmugalingam, Interpolation properties of Besov spaces defined on metric spaces, Math. Nachr. 283 (2010), 215-231.

[8] P. Hajłasz, Sobolev spaces on an arbitrary metric space, Potential Anal. 5 (1996), 403-415.

[9] -, A new characterization of the Sobolev space, Studia Math. 159 (2003), 263-275.

[10] P. Hajłasz and P. Koskela, Sobolev met Poincaré, Mem. Amer. Math. Soc. 145 (2000), no. 688, 101 pp.

[11] D. D. Haroske and C. Schneider, Besov spaces with positive smoothness on $\mathbb{R}^{n}$, embeddings and growth envelopes, J. Approx. Theory 161 (2009), 723-747.

[12] L. I. Hedberg and Yu. Netrusov, An axiomatic approach to function spaces, spectral synthesis, and Luzin approximation, Mem. Amer. Math. Soc. 188 (2007), no. 882, $97 \mathrm{pp}$.

[13] J. Heinonen, Lectures on Analysis on Metric Spaces, Springer, New York, 2001.

[14] M. Kabanava, Function spaces on the Koch curve, J. Funct. Spaces Appl., to appear. 
[15] M. Kabanava, Function spaces on the snowflake, in: Function Spaces IX (Kraków, 2009), Banach Center Publ., to appear.

[16] S. Keith and X. Zhong, The Poincaré inequality is an open end condition, Ann. of Math. 167 (2008), 575-599.

[17] P. Koskela and E. Saksman, Pointwise characterizations of Hardy-Sobolev functions, Math. Res. Lett. 15 (2008), 727-744.

[18] P. Koskela, D. Yang and Y. Zhou, A characterization of Hajtasz-Sobolev and Triebel-Lizorkin spaces via grand Littlewood-Paley functions, J. Funct. Anal. 258 (2010), 2637-2661.

[19] - - - - , Pointwise characterizations of Besov and Triebel-Lizorkin spaces and quasiconformal mappings, preprint, Beijing, 2010; arXiv:1004.5507.

[20] P. Malliavin, Integration and Probability, Springer, New York, 1995.

[21] D. Müller and D. Yang, A difference characterization of Besov and Triebel-Lizorkin spaces on RD-spaces, Forum Math. 21 (2009), 259-298.

[22] Ju. V. Netrusov, Sets of singularities of functions on spaces of Besov and LizorkinTriebel type, Trudy Mat. Inst. Steklov. 187 (1989), 162-177 (in Russian); English transl.: Proc. Steklov Inst. Math. 187 (1990), 185-203.

[23] S. M. Nikol'skiı̌, Approximation of Functions of Several Variables and Imbedding Theorems, 2nd ed., Nauka, Moscow, 1977; 1st ed. 1969 (in Russian); English transl.: Springer, New York, 1975.

[24] J. Peetre, A remark on Sobolev spaces. The case $0<p<1$, J. Approx. Theory 13 (1975), 218-228.

[25] C. Schneider, Spaces of Sobolev type with positive smoothness on $\mathbb{R}^{n}$, embeddings and growth envelopes, J. Funct. Spaces Appl. 7 (2009), 251-288.

[26] -, On dilation operators in Besov spaces, Rev. Mat. Complut. 22 (2009), 111-128.

[27] C. Schneider and J. Vybíral, On dilation operators in Triebel-Lizorkin spaces, Funct. Approx. Comment. Math. 41 (2009), 139-162.

[28] W. Sickel and H. Triebel, Hölder inequalities and sharp embeddings in function spaces of $B_{p q}^{s}$ and $F_{p q}^{s}$ type, Z. Anal. Anwendungen 14 (1995), 105-140.

[29] S. L. Sobolev, Some Applications of Functional Analysis in Mathematical Physics, Izdat. Leningrad. Gos. Univ., Leningrad, 1950 (in Russian); English transl.: Amer. Math. Soc., Providence, 1991.

[30] E. M. Stein, Singular Integrals and Differentiability Properties of Functions, Princeton Univ. Press, Princeton, 1970.

[31] H. Triebel, Theory of Function Spaces II, Birkhäuser, Basel, 1992.

[32] - , The Structure of Functions, Birkhäuser, Basel, 2001.

[33] -, Spaces on sets, Uspekhi Mat. Nauk 60 (2005), no. 6, 187-206 (in Russian); English transl.: Russian Math. Surveys 60 (2005), 1195-1215.

[34] —, Theory of Function Spaces III, Birkhäuser, Basel, 2006.

[35] - Function Spaces and Wavelets on Domains, Eur. Math. Soc. Publ. House, Zürich, 2008.

[36] -, Bases in Function Spaces, Sampling, Discrepancy, Numerical Integration, Eur. Math. Soc. Publ. House, Zürich, 2010.

[37] J. Vybíral, On dilation operators and sampling numbers, J. Funct. Spaces Appl. 6 (2008), 17-46.

[38] D. Yang, New characterizations of Hajłasz-Sobolev spaces on metric spaces, Sci. China Ser. A 46 (2003), 675-689.

[39] W. P. Ziemer, Weakly Differentiable Functions, Springer, New York, 1989. 
Hans Triebel

Mathematisches Institut

Fakultät für Mathematik und Informatik

Friedrich-Schiller-Universität Jena

07737 Jena, Germany

E-mail: hans.triebel@uni-jena.de

Received July 25, 2010

Revised version September 11, 2010 